\title{
María Flores-Fernández \\ Identités de Pandore : à la rencontre du paysage comme image de soi
}

\section{PANDORA'S IDENTITIES: DISCOVERING}

THE LANDSCAPE AS A SELF-IMAGE

Abstract: Considering the postmodern theoretical context of Maxine SheetsJohnstone's corporeal turn and la pensée paysagère ("landscaping thought") in Augustin Berque, the myth of Pandora's box is defined here as the self-image of landscape through the ecofeminism of Françoise d'Eaubonne (1974) and the identification of women to nature by Carolyn Merchant (1980). This perspective describes how ecofeminism, landscape philosophy and the imaginary of the body introduce the mutable nature of literary myths and, more precisely, the cosmogonic Pandora's identities assimilated to the archetypal image of the landscape-woman: from Eve-Pandora as femme fatale, the first woman, to Gaia-Pandora, bearer of hope.

Keywords: Structural Anthropology; Ecofeminism; Pandora; Geographical Imaginaries; Landscape; Myth-analysis; Mythcriticism.

\section{MARÍA FLORES-FERNÁNDEZ \\ Université de Grenade, Espagne mflores@ugr.es}

DOI: $10.24193 /$ cechinox.2021.40.20
Au même instant le tonnerre gronde; l'éclair sillonne la nue, et la foudre est tombée non loin de Pandore sur de beaux arbres quelle a réduits en cendres. (Charles Chaisneau, Pandore, poème en trois chants, $1808^{1}$ )

\section{Introduction}

P uisque l'imaginaire féminin se veut performatif ${ }^{2}$ tant dans le décadentisme que dans la postmodernité, il est essentiel d'étudier l'émergence des représentations symboliques du corps de la femme de la part d'auteurs qui ont défini des autoimages dans le paysage par le biais d'outils narratifs dans plusieurs domaines, comme l'art et l'écriture. S'intéresser aux identités féminines et à l'environnement, ainsi qu'aux formes contemporaines d'écritures écoféministes, répond à l'émergence des discours en lien avec le domaine paysager qui, non seulement pensent leur rapport au milieu psycho-géographique - l'archetope (Lévy, 2014) -, mais aussi à la " psychohistoire » (Neumann, 1953), tout en donnant la prééminence au féminin. Les études abordées antérieurement approfondissent une idée de Pandore comme le mal 
destiné aux hommes, ainsi que la notion de nature et paysage en lien avec l'art et la littérature (Collot, 2005 ; Roger, 1997). Cependant, il est nécessaire de proposer une nouvelle lecture de la corrélation entre le mythe et le paysage au croisement de la déesse. Notre étude s'articule autour de la symbolique associée à cette rencontre, en se concentrant principalement sur la figure de Pandore ainsi que sur la réactivation des invariantes universels en tant qu'image archétypale de la femme au caractère créateur, médiateur et transformateur. Dans les pages qui suivent, nous tenterons, tout en privilégiant la méthode durandienne, de mettre en évidence les signifiants associés à cette image symbolique afin de comprendre les significations projetées par la psyché humaine dans la mythologie, l'anthropologie, la littérature et la création artistique depuis le décadentisme jusqu'à nos jours.

À partir du substrat théorique résultant $\mathrm{du}$ « tournant corporel » (SheetsJohnstone, 2009) et de la "pensée paysagère » (Berque, 2008) de la postmodernité, cette étude se propose de définir l'image de soi en relation avec le corps féminin et le « paysage incarné » (Chiron, 2009) ; un tournant du corps féminin comme paysage. L'écoféminisme, introduit par Françoise d'Eaubonne en 1974, désigne l'assimilation de la femme à la nature et a été consolidé et reformulé par d'autres auteurs - comme Carolyn Merchant (1980) - au $\mathrm{XX}^{\mathrm{e}}$ siècle. Sur cette base théorique, Pandore en tant que femme-paysage s'avère à la fois un mythe fondateur et un mythe en train de naître, à notre avis, en raison de trois traits spécifiques et prédominants de son identité : Pandore comme femme fatale au décadentisme (Verlant, 2006),
Pandore en tant que médiatrice en modernité (Illich, 1971) et Pandore-Gaia en postmodernité (Lévêque, 1988 ; Maffesoli, 2014). Comment l'écoféminisme, la philosophie du paysage et l'imaginaire du corps permettent-ils de revenir sur la nature changeante des mythes littéraires et, plus précisément, sur une Pandore cosmogonique à redéfinir ? En quoi les différents traits de l'identité de Pandore relèvent l'image archétypique de la femme-paysage comme image de soi ? Cet article propose de repenser le statut de cette figure mythologique dans le processus d'individuation à travers l'étude des images-symboles, l'expérience de soi dans l'espace et l'image psychocorporelle. Le but est, par conséquent, d'analyser la dimension mythopoïétique des liturgies modernes au sens où elles sont productrices du mythe de Pandore et de leurs images transculturelles - la triade Ève-Sophia-Gaia -, des représentations intériorisées dans l'esprit et devenues les guides de la pratique de la postmodernité.

\section{Pandore comme femme fatale : le mythe d'origine}

Dour comprendre la transition des trois figures symboliques de Pandore et leur lien avec le paysage, il est d'abord nécessaire de revenir sur les réécritures du mythe au XVI ${ }^{\mathrm{e}}$ siècle, comme Eva Prima Pandora de Jean Cousin l'Ancien, le premier nu de la Renaissance française. Cette image témoigne du rôle que jouent les images et les métaphores dominantes dans la formation des valeurs et des comportements culturellement acceptés à l'égard des femmes et de la nature, spécialement dans l'assimilation du corps de la femme à un espace géographique. Dans le cas de cette 
peinture énigmatique, le lien se produit par une double identité : il s'agit de deux paysages opposés et divisés par un fleuve ; l'un sauvage - incarnant l'image d'Ève -, l'autre apprivoisé et constituant une cité antique - renvoyant à Pandore. D'après Dora et Erwin Panofsky, « au milieu du $\mathrm{XV}^{\mathrm{e}}$ siècle la comparaison, et même l'identification, entre une grande ville et Pandore, semble avoir été plus ou moins courant dans l'humanisme français ${ }^{3}$ ». C'est le cas de la Rome-Pandore de Joachim du Belley dans les Antiquitez de Rome, où le paysage de la capitale italienne "belle et pécheresse ${ }^{4}$ montre la personnification de cette figure mythologique. En outre, celle-ci incarne la ville de Paris en tant que «nouvelle Pandore vêtue en nymphe » dans l'une des représentations en relief créée par Jean Cousin et Jean Goujon pour ornementer l'arc triomphal qui a accueilli Henri II en 1549. Les deux premières femmes, la biblique et la mythique, sont à la fois opposées et unifiées par leur symbolique universelle : elles déploient une séduction fatale tout en incitant l'homme à succomber au péché. En effet, c'est par la plume du mythographe Henri Estienne que la puissance séductrice et destructrice de l'archétype de la « femme fatale " chez Ève et Pandore est célébrée dans « la création d'Adam et Ève, et de leur transgression, en prenant le premier homme forgé par Prométhée, pour Adam, et ceste jeune fille, nommée Pandore, pour Ève, laquelle amenée à Adam, fut cause de son mal $»^{5}$. Comme dans la Genèse, où Evve est coupable de l'exclusion du paradis, c'est avec Pandore que commence l'ordre séculier du monde ; cependant, notre regard se penche particulièrement sur la représentation de Pandore en tant que femme-paysage à l'époque décadente en raison de l'émergence de l'archétype de la femme fatale, tel que Maurice Scève l'exprime dans sa poésie : «qu'au premier œil mon âme l'adora, comme de tous la délation et de moi seul fatale Pandora $"^{6}$. Plus tard - dans le modernisme et le postmodernisme -, cette représentation donnera lieu à une lecture syncrétique et écoféministe de ce mythe d'origine.

Dans La poétique de la rêverie, Gaston Bachelard montre comment au moment de la genèse de la culture " les mythes sortaient de terre, ouvraient la terre pour qu'avec l'œil de ses lacs elle regarde le ciel $»^{7}$. D'après lui, «l'homme exprimait la terre, le ciel, les eaux. L'homme était la parole de ce macro-anthropos qu'est le corps monstrueux de la terre ${ }^{8}$. C'est là que le corps de la terre, à savoir le paysage - le macrocosme -, se fait corps de femme - microcosme -, et celui-ci ne fait qu'un avec l'univers. Articuler le singulier et l'universel, deux polarités se trouvant au sein de l'identité, répond à une dimension totémique et créatrice de la femme-paysage qui accueille des archétypes pluriels liés aux quatre éléments de la nature $^{9}$. De même, tel que le souligne Csilla Kemenczei, analyste jungienne et créatrice de la méthode d'Analyse des Mythes Corporels $(\mathrm{AMC})^{10}$, « dans son état primordial de Terre mère matricielle, la femme représente la toute-puissance indifférenciée ${ }^{11}$. Cette relation s'appuie sur le constat que c'est à travers la découverte de cette puissance qu' " émerge alors une conscience qui est incarnée par la Déesse Mère. C'est l'instant où la conscience rencontre la nature et la déesse sort des viscères de la terre $»^{12}$. Ainsi, la relation entre la femme et la nature - particulièrement avec le paysage - introduit un féminin sacré s'appuyant sur l'image cosmique de la femme 
et les mythes paysagers gréco-romains de Pandore et Déméter, déesses de la terre féconde, de la maternité, de la mort et de la résurrection. Parmi les multiples significations de « mère » enregistrées par Chevalier et Gheerbrant, « la mer et la terre sont les symboles du corps maternel " (Chevalier et Gheerbrant, 1991 : 165). Dans ce sens, la mère-terre prend la valeur d'un archétype et « la première forme que prend l'expérience de l'anima pour l'individu est la mère, c'est-à-dire l'inconscient ", et par conséquent, l'image de soi. Dans la même ligne de pensée, Erich Neumann examine l'évolution des significations de l'archétype de la Terre depuis le Moyen-Âge ${ }^{13}$ jusqu'à nos jours tout en concluant que la réémergence récente de l'archétype de la terre - et son resurgissement à la période décadente d'après notre hypothèse de même que le lien avec la terre, est cruciale pour comprendre la pensée de l'homme moderne. La terre et le corps maternel se sont donc confondus dans un tout, attirant la société à participer à un culte décadent et moderne des représentations du féminin. Et ce lien avec le féminin témoigne l'amalgame de données psychologiques, littéraires et artistiques relatifs au culte de la femme-terre, Grande Déesse ou Terre-mère. En effet, les identités créatrices féminines concernent une capacité à générer, à porter fruit, non seulement sur le plan biologique, mais aussi symbolique et archétypologique.

Étant donné que les archétypes sont des « matrices inconscientes héritées

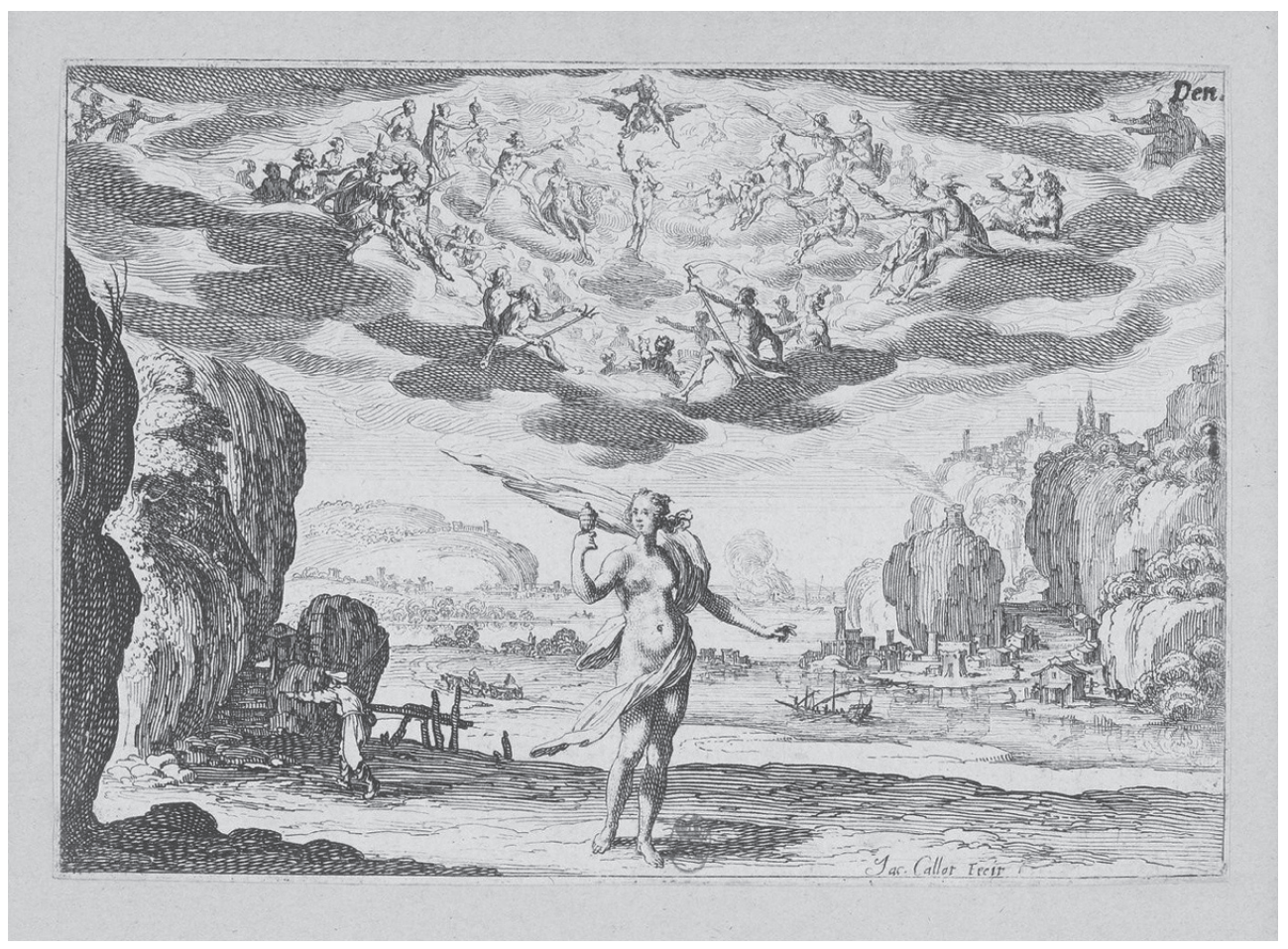

Illustration 1. Pandore, gravure de Jacques Callot, 1626. Bibliothèque Nationale de France 
génétiquement ${ }^{14}$, ils accueillent des pluralités à l'intérieur d'un bassin sémantique, en l'occurrence, déterminé par le progrès

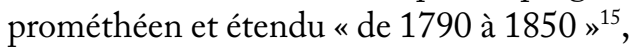
d'après la thèse de Gilbert Durand. C'est lors de cette période isotopique, composée d'invariants transculturels, que Pandore, déesse apparemment destructrice, idéal de beauté, de paix et d'espérance, médiatrice entre les hommes, commence à réactiver ses traits multiples sous différentes manières.

Pandore est une figure féminine remarquable de l'héritage antique : elle apparaît, aux dires de Jean-Pierre Vernant, comme « la première femme, l'ancêtre de toutes les femmes ${ }^{16}$, c'est-à-dire la Mère de l'humanité, dans un mythe étant le premier dans la pensée occidentale à s'organiser autour d'une femme "modelée à la semblance d'une vierge humaine qui n'existe pas encore et dont elle va précisément constituer le prototype ${ }^{17}$. Ainsi, elle devient " l'équivalent d'un eidôlon : un double, tout à fait semblable lui aussi à un être véritable, mais vide, inconsistant, insaisissable, absent dans sa présence ${ }^{18}$. Le récit mythico-religieux sur l'énigme de création de la première femme, repose sur l'aliénation d'Êve et Pandore en tant qu'incarnation du beau et du mal - kalòn kakòn, un beau mal, selon la Théogonie d'Hésiode $^{19}-\mathrm{du}$ féminin universel qui règne dans le monde de la nature, du péché et de l'instinct. D'après Florence Godeau, spécialiste en littérature comparée, elle a été décrite comme une « créature maléfique et rusée » souvent identifiée comme Ève dans les réécritures du mythe hésiodique, « ou, bien au contraire, notamment par le biais de la métamorphose goethéenne, comme la gracieuse dispensatrice de ces valeurs prométhéennes que promouvra davantage le Romantisme $»^{20}$. De surcroît, en tant quêtre primordial cyclique, elle s'inscrit dans le régime nocturne, dans la dimension syncrétique de l'image au sens durandien. Selon la pensée de Gilbert Durand, dans la matrice unidimensionnelle et linéaire du régime diurne présente dans le récit biblique, la nature et la femme sont « connaturelles à l'animal » ${ }^{21}$ et à la fatalité, à ce qu'il faut dominer. Or, dans le régime nocturne - où le temps est cyclique - , c'est le ventre de la mère réceptive, le réceptacle, le creux, le culte universel du féminin qui renvoie à l'image du " paysage incarné ${ }^{22}$. Ève, mère de tous les êtres vivants, porte depuis les temps anciens le lourd fardeau d'avoir succombé à la tentation, de l'expulsion du paradis et de la chute dans la maladie, la douleur et la mort. Cette féminisation de la chute morale et du péché originel, en lien avec le corps et la nature par son caractère cyclique, est repris par Durand, car « la femme, d'impure qu'elle était par le sang menstruel, devient responsable de la faute originelle » et dans d'autres traditions "c'est pour expier une faute que les femmes sont réglées $»^{23}$. Cette chute morale, d'après lui, « nourrit également le mythe grec de Pandore ${ }^{24}$ tout en suivant un système d'analogies du corps avec les effets cycliques de la nature sur le paysage, et qui, pourtant, selon notre hypothèse, a toujours appartenu aux grandes déesses ancestrales du cycle naturel et des mystères de la vie et de la mort. Au fait, dans l'imaginaire de la descente, parmi les symboles nyctomorphes introduits par Durand, il se trouve la femme fatale, la lune noire, la mort liée au féminin avec l'animal, ainsi que le sang menstruel et le péché originel ${ }^{25}$. Le voyage au fond de la terre implique la valorisation 
de substances telles que la boue et le limon, avec lesquelles le corps de Pandore est fait, tel que Hésiode l'exprime dans Les Travaux et les jours :

Il dit commande à l'illustre Héphaïstos de tremper d'eau un peu de terre sans tarder, d'y mettre la voix et les forces d'un être humain et d'en former, à l'image des déesses immortelles, un beau corps aimable de vierge ; $(\ldots)$ à cette femme il donne le nom de Pandore parce que ce sont tous les habitants de l'Olympe qui, avec ce présent, font présent du malheur aux hommes ${ }^{26}$.

Cependant, quant au potentiel créatif de la figure de Pandore, il est à noter que l'utilisation de la boue ou de l'argile est définie par Bachelard comme " un limon primitif, apte à recevoir et à garder la forme de toute chose ${ }^{27}$. À la lumière de la poétique de la matière bachelardienne, l'argile est la manifestation sensible de la "pâte idéale » ou « la pâte parfaite $»^{28}$, l'union de la terre et de l'eau, une matière imaginaire modulant la pensée et l'identité féminine. Dans le mythe de Prométhée, le résultat est une figure de fascination, conçue à l'image de dieux, de même que dans les Métamorphoses d'Ovide, où la terre "naguère encore grossière et indistincte, prit forme et se modela en figures nouvelles d'êtres humains $»^{29}$. Lunion de ces matières présentes dans le milieu naturel renvoie à la Pandore qui apparaît dans le poème érotique de Charles-Pierre Colardeau, Les Hommes de Prométhée (1774), où son corps est présenté comme un paysage enchanté ${ }^{30}$, de même que la Pandore-paysage symboliste d'Odilon Redon.
$\mathrm{Au}$ cours du XIX ${ }^{\mathrm{e}}$ siècle, les thèmes de la corporéité et du paysage sont repris sous diverses formes et deviennent de plus en plus récurrents. D'un côté, l'hypothèse corporelle est claire dans la philosophie du romantisme et dans les représentations de l'archétype de la femme fatale pendant le décadentisme. D’une certaine façon, l'esprit romantique - et parfois libérateur - du XIX e siècle s'est adapté à l'esprit de l'époque, comme dans la tradition symboliste anglaise de l'œuvre Pandora (1871) de Dante Gabriel Rossetti ${ }^{31}$ et Pandora (1896) de John Williams Waterhouse ${ }^{32}$. Pendant le romantisme, malgré le fait que les Pandores étaient décrites comme des femmes-fatales, Goethe ${ }^{33}$ et Balzac ${ }^{34}$ introduisent, par rapport à La Théogonie et Les Travaux et les jours de Hésiode, des variantes qui altèrent le sens originel du récit mythique. Avant l'ère d'un Dieu créateur et solaire, ce sont les déesses primordiales lunaires qui incarnent ce rôle dans toutes les grandes civilisations. Cependant, dans The Myth of the Goddess: Evolution of an Image, Anne Baring et Jules Cashford expliquent que c'est en raison du retournement du mythe d'Ėve que la Grande Déesse est dépourvue de son double rôle ancestral. Lorsque l'Homme décide de séparer le bien du mal et le Créateur de la création, il fait le choix de ne retenir de l'archétype féminin que son pouvoir de destruction. Il renie donc l'autre fonction que la Déesse mère avait jusqu'alors exercée : donner vie à toute création et accorder la libération et l'immortalité. D'un autre côté, aux dires de Panofsky, Pandore, "inaugure une série d'œuvres du XIX ${ }^{\mathrm{e}}$ siècle dans lesquelles les noms et les attributs de Pandore ne sont guère qu'un prétexte à montrer une charmante nudité ou semi-nudité féminine $»^{35}$. 
Cette nudité est transposée au paysage à travers la mutation « d'un pays (asexué) en paysage (érotisé) ${ }^{36}$. Selon Alain Roger, « il y a du pays, mais des paysages, comme il y a de la nudité et des nus ${ }^{37}$. Ce phénomène orchestré par l'artialisation des corps et la féminisation du paysage, ainsi que par la notion de nature comme création à partir de notre perception, est à cerner à partir du concept d'identité en tant que construction et moyen de s'autoreprésenter. Tel que l'entend Michel Collot, « la nature est à la fois belle et fatale, c'est qu'elle est femme ${ }^{38}$ et en ce sens, ce n'est pas la femme et le paysage qui s'autodéfinissent, mais l'homme qui les détermine par le biais de l'artialisation de la nature, terme qui signifie transformer la nature par le biais de l'art. Alain Roger affirme, tout en prenant comme exemple le corps de la femme, qu' « à l'instar de la nudité féminine, qui n'est jugée belle qu'à travers un $\mathrm{Nu}$, variable selon les cultures, un lieu naturel n'est esthétiquement perçu qu'à travers un Paysage " ${ }^{39}$. Cette idée sera reprise et développée à la fin du modernisme à partir de nouvelles identités de Pandore, comme expliqué ci-après.

\section{Pandore en tant que médiatrice : le mythe d'espoir}

Tévolution planétaire et l'effondrement sont marqués par « le retour d'Hermès " ${ }^{40}$, d'après Gilbert Durand, et sa prolon-

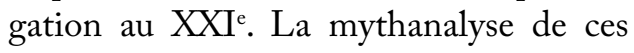
structures de l'imaginaire met en lumière l'antagonisme entre le mythe de Prométhée et un hermétisme patent, et aussi un aspect féminin intermédiaire : Pandore et son lien avec l'élément terre; on ne parle pas seulement du mythe de Dionysos ${ }^{41}$, précédé par un Prométhée promouvant la domination et l'exploitation de la nature à travers le développement des sciences et des techniques ${ }^{42}$. Chez Durand "le nouveau mythe de notre temps est celui du lien entre les différences, de la médiation entre prochain et lointain, celui des bornes, des limites qui définissent seules rencontres et carrefours ${ }^{43}$, à savoir le mythe d'Hermès. Dans ce sens, très proche aux « constellations d'images ${ }^{44}$ durandiennes, Ivan Illich, contemporain de Gilbert Durand et défini comme un " astronome de l'humain ", reste fidèle au mythe de Pandore, comme figure médiatrice, à côté du messager ailé. Sans citer ses sources, Illich remet en cause l'interprétation habituelle du récit mythique, car il résulte, en effet, qu'en ce temps de transition, c'est un mythe féminin qui commence à prendre le relais du mythe masculin de Prométhée. Cette métamorphose marquée par le passage d'un mythe à un autre, est représentée dans une sculpture insérée dans un milieu paysager qui apparaît dans l'entretien de Jean-Marie Domenach avec Ivan Illich, paru dans la série Un certain regard, le 19 mars 1972, à Paris. Cette sculpture montre une Pandore qui semble se réincarner dans les femmes fatales et décadentes. Celle-ci apparaît, dans toute la terreur de sa puissance corruptrice et destructrice, comme la Mère-Terre face à l'bybris l'excès et la démesure — de Prométhée. Loin de la misogynie présente au décadentisme, la nouvelle Pandore fait partie de ces femmes peintes dans la littérature et l'art symboliste, comme la Salomé de Gustave Moreau, et que nous découvrirons plus puissantes et moins menaçantes dans l'imaginaire masculin. Cette nouvelle identité a un rôle plus noble à remplir 
auprès de l'homme Épiméthée, paradoxalement grâce à un double aspect décrit dans la poésie hésiodique : «Elle montre l'ambivalence du feu, qui a donné à l'humanité un immense pouvoir, mais celui-ci peut tourner à son malheur, aussi bien qu'à son bonheur, selon que le désir des hommes sera droit ou pervers $»^{45}$. L'apparence de Pandore recouvre donc, d'après le regard masculin d'Hésiode, l'idée de danger et d'impureté attribuée à la femme, mais elle intègre aussi, chez d'autres penseurs comme Illich, l'écosystème et la nature " par la substitution de la pythia, d'un monde qui voyait Pandore comme tenant une caisse, par la vieille Mère-Terre que nous, les nouvelles générations, contemplons comme l'étoile bleue, avec nostalgie de la Lune ${ }^{46}$. Dotée par les dieux d'une voix et parée de tous les dons - beauté, intelligence, habileté -, mais aussi pourvue de la sagesse de Sophia, elle est menée par Hermès jusqu'au frère de Prométhée, Épiméthée. Celui-ci reçoit volontiers le cadeau, malgré les avertissements de son frère. Et en effet, à peine Épiméthée a-t-il accepté le cadeau que la femme ouvre la fameuse boîte - dans la tradition d'origine, il s'agit d'un pithos $\left(\pi i \theta_{0}\right)^{47}$, une grande jarre en argile - boîte d'où sortent tous les maux sur la terre ; d'après les versions de Hésiode, tous les maux y sont sortis, sauf l'espoir, un don qui ouvre la réinterprétation du mythe. À cet égard, Ivan Illich soutient que toute la mythologie classique dès lors est orientée vers l'avenir, vers l'effort de mettre dans une boîte les maux que la Pandore classique a laissés s'échapper. Il reprend ainsi une version du mythe antérieure à Hésiode, dans laquelle Pandore est " celle que tout le donne ", une divinité matriarcale grecque. Pandore est la source de tous les dons que la terre offre et Epiméthée, l'homme qui fait honneur à la femme en se donnant à elle. Dans la nouvelle perspective, ce qui est conservé dans la jarre n'est pas une attente, mais un espoir, ici compris comme confiance dans lordre providentiel de la terre, dans la bonté de la nature - et aussi dans sa capacité à se rebeller contre l'oppression -, tandis que l'attente signifie confiance dans des résultats qui sont planifiés et contrôlés par l'homme, au sens prométhéen. Ainsi, le mythe de Prométhée se verra éclipsé au $\mathrm{XX}^{\mathrm{e}}$ siècle, par le surgissement d'une nouvelle identité de Pandore, liée à Hermès. D'après Jean-Pierre Vernant, « Pandora s'est introduite chez les hommes : elle est la femme d'Épiméthée, la première épouse ${ }^{48}$. Épiméthée a défendu par ses actes un mode de vie différent basé sur le respect de l'environnement, l'amour du prochain et l'espoir d'un autre monde. Dans l'histoire de l'Occident et du capitalisme, au sens plus large, Pandora symbolise la femme et la nature qui donnent la vie et en même temps la mort. Sur ce dernier point, il faut considérer attentivement une nouvelle mythologie contestataire qui se forge sous la plume de Erich Neumann, Blanca Solares ${ }^{49}$ ou Naomi Goldenberg ${ }^{50}$, tout en démystifiant le mythe patriarcal de Prométhée du $\mathrm{XIX}^{\mathrm{e}}$ siècle. Celui-ci perd ses mythèmes, en intègre d'autres et finit par se muer en Pandore et Hermès, deux mythes qui se rencontrent dans un même bassin sémantique sétendant vers la postmodernité en tant que médiateurs et porteurs d'espoir. Selon cette perspective, Prométhée est le seul être capable de détruire le monde d'Épiméthée l'écosystème - et Pandore - la nature, la femme-paysage - la seule à le libérer. 


\section{L'archétopos de Géa-Pandore : un mythe en train de naître}

'image de soi en tant que paysage inté-
rieur, qui est souvent comprise comme étant subjective, se matérialise par le biais de la représentation du corps en lien avec la nature, comme le note Victor Hugo dans Les Contemplations $^{51}$. À part la pensée romantique, ce rapport renvoie aujourd'hui à un phénomène d'identification et d'appropriation du paysage à travers l'image de soi au féminin et l'ensemble de représentations du corps qui témoigne d'un système d'isotopies. Ainsi, la pluralité des identités de Pandore converge-t-elle dans la résurgence du mythe comme une voie de production d'une identité essentielle au corps de la femme. L'une des nouveautés les plus connues et même les plus populaires de la philosophie et, en effet, de la pensée du $X^{\text {e }}$ siècle est ce que nous pouvons appeler le « bodily turn », c'està-dire la place de la corporalité humaine dans les discours littéraires et artistiques ; celle-ci est un thème pertinent de réflexion et de débats théoriques ; par exemple, pour Maxine Sheets-Johnstone le corps est « une simple matérialité dans le mouvement ${ }^{52}$. En revenant sur la sculpture parisienne de Pandore, Illich met en évidence cette dimension corporelle cinétique et cyclique dans la capacité nourricière de la femme-terre qui est donneuse de tout - en tant que divinité génératrice de vie -, et à la fois destructrice et libératrice : "Sur son sexe, tu trouves le serpent avec une tête de mort. C'est la femme dont la matrice a été envoyée dans ses mains et devenue une terre libre $"^{53}$. De même que dans l'œuvre Le Deluge (1733) de Bernard Picart, Pandore porte l'attribut sur son utérus ; ce serpent renvoie, à son tour, à la Gaïa-Pandora, non seulement en tant

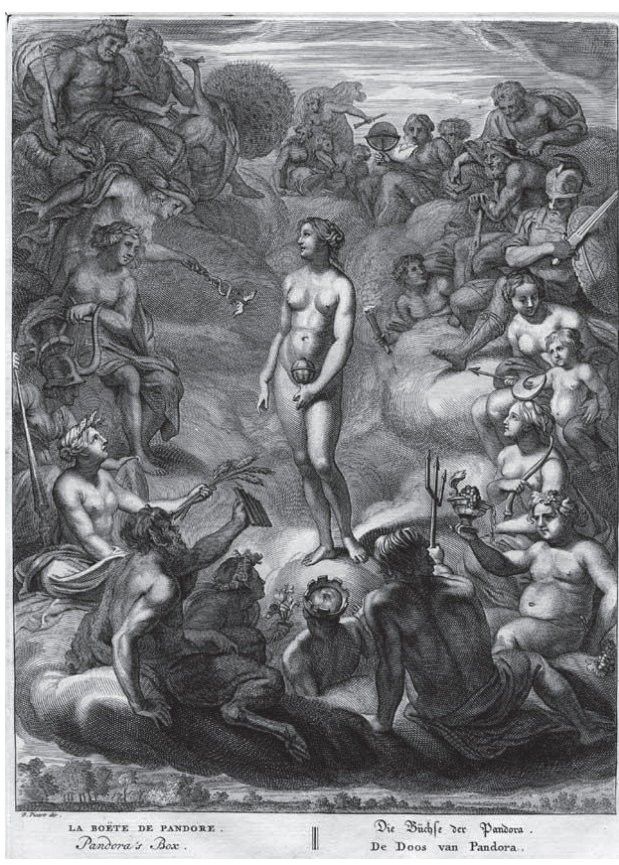

Illustration 2. Bernard Picart, plaque La boîte de Pandore, 1733

que médiatrice, symbole du kairos comme "région médiatrice de l'être comme mixte $»^{54}$, mais comme " archétopos» ou " archétype psycho-géographique ». C'est Bertrand Lévy qui introduit une dimension géographique de l'archétype et qui atteste que le paysage « contient et reflète des archétypes ${ }^{55}$.

La perspective introduite par Ivan Illich dans son entretien avec Jean-Marie Domenach, où Pandore devient Gaïa, la Mère-Terre, nous a conduit à repenser la Vierge-Marie et Tonantzi Iztaccihuatl comme des Pandores médiatrices, au sens de quatre niveaux de l'Anima junguienne. D'après Jean-Louis Benoît, la Vierge-Marie " est reine, mais avant, mère » et reprend la formule de saint Bernard qui la définit en tant que " médiatrice auprès du médiateur ${ }^{56}$. Une remarque s'impose toutefois. Dans leur forme dégénérée comme dispensatrices 
de grâces, ces deux déesses coexistent avec difficulté au Mexique, étant donné que lors de la Conquête et l'extension de la culture occidentale, les symboles de la Vierge Marie, de la Gaïa Hellénisée et la Tonantzin néolithique des Aztèques ont été confondus. Cependant, ces déesses font hommage à la maternité et dans quelques cultures qui peuplaient la Méso-Amérique, Coatlicue aussi appelée Tonantzin, est la mère de la plupart des dieux aztèques, la déesse de la fertilité et de la terre, la force fécondatrice de la nature. En outre, elle est « celle qui porte une jupe aux serpents ${ }^{57}$ et représente la vie et la mort, ce qui expliquerait la broderie de deux serpents sur la robe de la sculpture de Pandore montrée par Ivan Illich.

La dernière identité de Pandore créée par l'appropriation des caractères de Sophia comme la grand-mère, symbole de transformation spirituelle et symbole féminin d'intersection " théophanie féminine par le biais de laquelle la lumière primordiale se fait enfermer dans le monde sensible " et celle qui assure "le passage de la totalité manifestée du néant primordial à la totalité du cosmos $\aleph^{58}$. Elle réunit dans une identité individuelle la pluralité de ses images. Mais il est évident que les représentations de la déesse Sophia et celles de Pandore se sont dissipées - tout comme la nature et le voile d'Isis qui « aiment à se voiler ${ }^{59}, p h u-$ sis kruptesthai philei, aphorisme que Pierre Hadot emprunte à Héraclite - dans l'histoire de la philosophie et la religion. D'après Le Dictionnaire traditionnel des symboles de Juan Eduardo Cirlot, la pomme d'Ève, de même que la jarre de Pandore, «de forme presque sphérique ", représente " une totalité ; c'est un symbole des désirs terrestres, de leur déchaînement ", ou similaire à la curiosité, représente « la soif de connaissance, zone seulement intermédiaire entre les désirs terrestres et ceux de la pure et véritable spiritualité ${ }^{60}$. De même que Sophia, Ève et Pandore, Isis se définit comme « la terre ou la nature qui est sous le soleil. C'est la raison pour laquelle le corps tout entier de la déesse est hérissé d'une multitude de seins serrés les uns contre les autres, parce que l'ensemble des choses est nourri par la terre ou par la nature ${ }^{61}$, ce qui renvoie à l'assimilation du corps à l'orographie du paysage, à la concentration de plusieurs identités du féminin dans un seul corps qui devient paysage.

Très proche de la mythologie, l'écofeminisme est structuré sur la base d'une position éthique : une autodéfinition de l'identité des femmes fondée sur l'expansion de la justice dans un monde égalitaire et proche de la nature, idée également prônée par les romantiques. Ces aspects nous permettent de comprendre que l'écofeminisme n'implique pas seulement un tournant écologique du féminisme, mais aussi une identité féministe de l'écologie et de la mythologie. Plus précisément, il doit tenir compte du rôle éminent des femmes dans l'équilibre écologique, de sorte que celle-ci doit alors appeler la femme à aspirer à un équilibre de son propre corps, à l'image de soi et son mode d'être-au-monde; voici la dimension "psycho physiologique " ${ }^{62}$ introduite par Durand, dans ce cas-là, par laquelle va opérer la lecture postmoderne du mythe de la jarre. Pour Illich, Pandore est une déesse de la terre, « dispensatrice de tous les dons ", dont l'origine remonte à l'époque patriarcale préclassique ; cette amnésie de l'espoir issue de la lecture du mythe de Pandore chez les Grecs classiques légitime la présomption de pouvoir renfermer les maux et inaugure la fin de l'époque prométhéenne, malgré le retour à la croissance industrielle et technologique et les limites 
des écosystèmes au présent. D'après la pensée D'Eaubonne, c'est à partir de la reprise de ces valeurs que pourra être envisagée " une société de démocratie directe, objectif toujours visé et toujours perdu par des révolutions qui ignorent la moitié $d u$ ciel et la totalité de l'environnement ${ }^{63}$, car « la planète mise au féminin reverdirait pour tous ${ }^{64}$. Avec l'oubli du mythe originel de Pandore se perd l'espoir dans les forces de la nature ; de plus, le processus historique symbolisé par le développement des techniques et des lois démarre. Prométhée - qui avait prévenu son frère Épiméthée de se méfier de Pandore s'affirme dans l'imaginaire collectif comme icône de la providence et de la connaissance humaine. Dès le déclin du mythe de Pandore, la « raison sensible " ${ }^{65}$ 'est soumise à la raison prométhéenne, ou plutôt, à l'éthos prométhéen qui a effacé l'espoir. Une possible analogie proche de la lecture mythocritique de la déesse comme symbole de l'écoféminisme est celle proposée par Jean-Pierre Vernant à partir du récit d'Hésiode : le corps féminin est comme le champ que le paysan fertilise, la femme donne la vie parce qu'elle porte les fruits de la semence de l'homme. Or, d'après l'historien, la femme donne aussi la mort parce quelle « absorbe toutes les richesses, toute la vitalité masculine ${ }^{66}$. En revanche, Carolyn Merchant considère que face à une nature vue jusqu'alors sous les traits d'un être nourricier, la révolution scientifique a fait prévaloir la nature comme désordre chaotique et destructeur qu'il faut dominer et contrôler ${ }^{67}$.

L'homme prométhéen a modifié l'équilibre de l'atmosphère et des écosystèmes, de sorte que le système économique actuel se limite à l'extraction des ressources de la nature et à l'exploitation de la femme. Nous pouvons remarquer que le mot " patrie » est

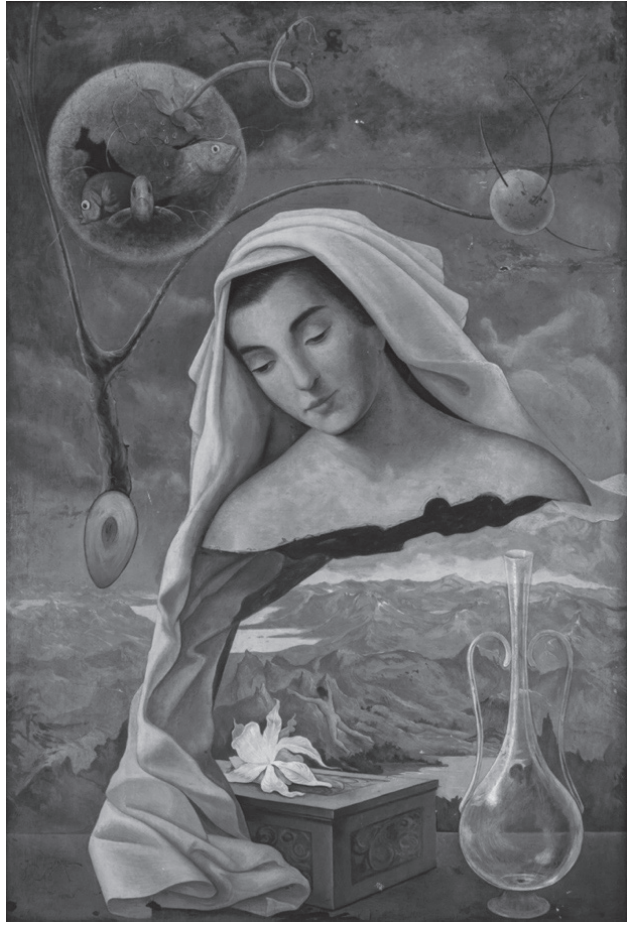

Illustration 3. Juan Gabriel Barceló Tomás, La caja de Pandora, 1963

construit sur l'idée que la terre est la terre du père. La patrie est d'abord le rappel constant d'une époque où seuls les hommes se définissaient dans leur appartenance au territoire. Cependant, au cours des cinquante dernières années, nous avons assisté à une tradition d'auteurs qui sont allés à la rencontre féminine du paysage, à la recherche d'un nouveau dialogue entre l'art, l'espace géographique et la nature. Ce nouveau mythe a été auparavant perméable aux discours artistiques postmodernes, comme c'est le cas de la représentation symbolique de la boîte de Pandore en 1963 par le peintre surréaliste espagnol Juan Gabriel Barceló Tomás. Il interprète le visage de sa femme Elisa, en imitant la déesse et le retour à une nouvelle identité du mythe en postmodernité. 
En définitive, il s'agit d'une représentation fondée sur l'idée qu'il n'est pas possible de séparer la nature et la culture - autoperception humaine -, car la notion de paysage est une production mentale construite à partir des couches de mémoire et des éléments naturels. L'ancienne identité de la nature en tant que mère nourricière, femme bienveillante qui pourvoyait aux besoins de l'humanité dans un univers ordonné et planifié, relie la terre féminine à une cosmologie organique qui, selon Merchant, est transformée en métaphore dominante de la révolution scientifique et technologique :

Tout comme l'utérus de la femme avait symboliquement cédé au forceps, l'utérus de la nature rxenfermait des secrets qui, grâce à la technologie, pouvaient être retirés de son emprise afin dêtre utilisés pour l'amélioration de la condition humaine ${ }^{68}$.

En ce sens, Ivan Illich, de même que Merchant et Françoise D'Eaubonne, se penchent sur la revendication des liens du féminin avec le vernaculaire, avec l'élément terre, ainsi que sur la défense des femmes, vu leur capacité biologique à créer et à donner vie. Le philosophe explique le passage de la conception archaïque de la nature inépuisable à celle de la nature asservie : « Je crois que l'histoire de Pandore est la meilleure histoire du détournement de l'homme du Delphos de la Terre, de l'interprétation des songes et des images vers l'homme qui planifie ${ }^{69}$. Elle cède à la curiosité qu'Hermès lui avait donnée et ouvre la boîte - le phytos ou le kypsele - contenant tous les maux, mais aussi, d'après une autre lecture du mythe, les dons destinés à l'humanité, les sciences et les arts. À ce sujet, Illich soutien une perspective écocritique du mythe, tout en présentant Pandore comme une nouvelle Gaïa, capable d'incarner une écosophie, une nouvelle culture planétaire basée sur une relation eurythmique et pacifique avec la nature, tel que Michel Maffesoli le prône à travers la notion du "holisme postmoderne ", une " conception globale d'un monde pluriel où une économie quelque peu étriquée tend à laisser la place à une écosophie autrement plus riche en ce qu'elle incarne tous les paramètre humains. Ce dont une raison sensible peut rendre compte $»^{70}$. Sous la forme d'un avertissement moral, Illich soutient que «si nous nous retournons vers Gaïa-Pandore et ne sommes pas capables de nouveau d'être conscients du langage des songes qu'elle peut interpréter, nous sommes condamnés $»^{71}$. Cette posture, en lien avec la raison sensible, rejoint la pensée paysagère d'Augustin Berque et sa médiance avec le corps. D'après lui, le paysage est une "identité entre le fait de penser et le fait qu'il y ait paysage $»^{72}$, c'està-dire une approche au soi géographique, et par conséquent, à l'hypothèse du paysage au féminin comme image de soi :

Concrètement incarné en un certain lieu, à une certaine époque, le sens profond du paysage nest autre que le rapport dynamique (le moment structurel) qui s'établit entre l'écoumène et la biosphère, comme entre la biosphère et la planète $[\ldots]$. Et la pensée paysagère, c'est la manière dont chaque être humain, de sa chair à ses actions, traduit cette médiance ${ }^{73}$.

Enfin, en ce qui concerne l'assimilation identitaire de Pandore à la Terre-Mère, nous 
retenons la prémisse de Pierre Lévêque, qui reprend la relecture du mythe " qui fait de Pandora une divinité de la Terre ou une épiclèse d'une telle divinité $~^{74}$, tout en soulignant son caractère polymorphe, car il met en avant l'identité d'une « Gè Pandora (notamment Vita herodotea Homeri, 18), d'une Rhéa Pandora (Diodore, III, 57, 2). Rhéa représentant une fécondité sans limite qui se heurte au refus dévorateur du père Cronos ${ }^{75}$. En ce sens, il est nécessaire de remonter au I ${ }^{\text {er }}$ siècle, où Philon d'Alexandrie, dans son œuvre De aeternitate mundi, utilise l'épithète " pandôros » - choisi par Hermès pour désigner la Pandore que Zeus a modelée de la terre et de l'eau - afin de construire l'allégorie de la terre et la maternité chez la déesse nourricière Gè, la TerreMère, une П $\alpha v \delta \omega ́ \rho \alpha$ « qui donne tout ce qui est utile ${ }^{76}$. Dans le même ordre d'idées, Gilbert Durand affirme que « la terre est notre mère primordiale, mais elle est aussi dans ses transformations, de l'agriculture aux industries métallurgiques, le fils du génie humain, le fils que toutes les mythologies donnent à l'homme par le mystérieux accouplement avec la mère " $" 77$ À l'égard de la symbolique féminine de l'élément terre, ce n'est pas la terre qui émoule la femme, comme souligne Platon, "pour concevoir et pour engendrer "; en réalité, c'est la femme qui « imite la terre ${ }^{78}$. En effet, d'après Csilla Kemenczei, " toute femme, aussi banale soit-elle, incarne la déesse, la femme absolue et la mère cosmique » (Kemenczei 2010).

\section{Conclusion : vers l'expression d'une}

\section{identité collective à lère postmoderne}

$\mathrm{L}$ e mythe de Pandore semble parcourir les trois derniers siècles sans s'essouffler. Étant donné l'ambivalence de la fatalité de Pandore et du lien qui l'unit à son pithos, ce trait a donné naissance à de nombreuses réécritures du mythe; cela vaut aussi bien pour les récits gréco-romains que pour les manifestations littéraires et artistiques plus récentes. Dans les transformations littéraires du mythe, telles qu'on les rencontre, par exemple, dans la poésie de la Renaissance ou dans la philosophie moderne, dans les lectures féministes de Carolyn Merchant, ainsi que dans les réflexions d'Ivan Illich, l'interprétation écoféministe du mythe reste dominante. D'abord redécouverte à la Renaissance, Pandore semble trouver une forme d'apothéose au $\mathrm{XIX}^{\mathrm{e}}$ siècle quand les poètes et les artistes s'approprient sa figure. Pandore permet ainsi de trouver une communauté culturelle de références et d'invariants mythémiques tout en offrant une constellation de résonnances sur les représentations de l'identité de la femme en lien avec la nature et de l'image de soi en tant que paysage. L'imaginaire catastrophique inhérent à la terre nourricière nous a permis de reprendre une lecture d'une nature transformée en divinité, ainsi que l'interprétation d'une identité diaïretique et destructrice implicite dans sa dimension cyclique. Enfin, si comme le souligne Gilbert Durand, « éternel féminin et sentiment de la nature vont de pair en littérature ${ }^{79}$ et dans les arts, cette bifurcation de l'imaginaire de la nature au croissement du féminin serait-elle alors une voie pour penser le monde dans sa totalité et introduire un modèle archétypale du dynamisme mythique de Pandore ? La philosophie du corps du $\mathrm{XX}^{\mathrm{e}}$ siècle a été un point de transition vers une nouvelle philosophie, d'une nouvelle ontologie à une nouvelle philosophie relevant le chiasme 
entre les archétypes identitaires du corps féminin et les archétopos paysagers. Grâce à l'archétypologie postmoderne (Braga 2019), il sera nécessaire de continuer à explorer une pensée et une pratique de la corporéité et de l'incarnation du paysage où les trois traits de l'identité de Pandore s'entremêlent et se complémentent.

\section{BibliogRAPHIE}

Gaston Bachelard, La Poétique de l'espace, Paris, PUF, 1957.

- - La Terre et les rêveries de la volonté, Paris, PUF, 1948.

Anne Baring, Jules Cashford, The Myth of the Goddess : Evolution of an Image, Londres, Penguin, 1991.

Augustin Berque, La pensée paysagère, Paris, Archibooks, 2008.

Corin Braga, Archétypologie postmoderne. D’Edipe à Umberto Eco, Paris, Honoré Champion Éditeur, 2019.

Pierre Brunel Dictionnaire des mythes littéraires, Paris, Éditions du Rocher, 1988.

Dictionnaire des mythes féminins. Paris, Éditions du Rocher, 2002.

Anna Caiozzo, Femmes médiatrices et ambivalentes: Mythes et imaginaires, Paris, Armand Colin, 2012.

Michel Cazenave, Visages du féminin sacré, Paris, Éditions Entrelacs, 2012.

Éliane Chiron, Paysages croisés. La part du corps, Paris, Publications de la Sorbonne, 2009.

Juan Eduardo Cirlot, Diccionario de simbolos tradicionales, Barcelone, Luis Miracle, 1958.

Michel Collot, La pensée-paysage. Philosophie, arts, littérature, Arles, Actes, 2011.

- - Paysage et Poésie, du romantisme à nos jours, Paris, José Corti, 2005.

Françoise D'Eaubonne, Le féminisme ou la mort, Paris, Pierre Horay, 1974.

- Écologie et féminisme : Révolution ou mutation ?, Paris, Libre \& Solidaire, 1978.

Gilbert Durand, Les Structures Anthropologiques de l'imaginaire : introduction à l'archétypologie générale, Paris, Dunod. 2016.

- - «Les mythèmes du décadentisme », in Décadence et Apocalypse, Cahiers du C.R.I.S.M., Dijon, Université de Bourgogne, 1986, p. 3-16.

- - Figures mythiques et visages de l'ceuvre : de la mythocritique à la mythanalyse, Paris, Dunod, 1992.

- - Introduction à la mythodologie. Mythes et sociétés, Paris, Albin Michel, 1996.

- - Champs de l'imaginaire. Textes réunis par D. Chauvin, Grenoble, Ellug, 1996.

Florence Godeau, «Le vaisseau Pandore : histoire d'une traversée, de l'Europe vers l'Amérique (sur Henry James, Pandora, 1884) », Hg. von Heinz-Peter Preußer, Françoise Rétif und Juliane Rytz. Pandora. Zur mythischen Genealogie der Frau. Pandore et la généalogie mythique de la femme, Seiten und 16 Abbildungen auf 8 Tafeln, 2012, p. 175-184.

Patrice Guillamaud, "L'essence du kairos » in Revue des Études Anciennes, Tome 90, 1988, no 3-4, p. 359-371.

Henri Estienne, L'introduction au Traité de la conformité des merveilles anciennes avec les modernes, Paris, 1566.

Pierre Hadot, Le voile d'Isis : Essai sur l'histoire de l'idée de Nature, Paris, Folio, 2004.

Hésiode, Théogonie, Les Travaux et les jours, Le Bouclier, traduit par Paul Mazon, Paris, Les Belles Lettres, «Collection des Universités de France », 1946.

Carl Gustav Jung, Métamorphoses de l'âme et ses symboles, Genève, Librairie de l'Université, Georg \& Cie, 1967

Csilla Kemenczei, Le féminin de la femme. "Rencontre Féminin-Masculin, regards croisés : MarieLouise von Franz - Pierre Solié ». France : Association Autour de Marie-Louise von Franz, 2010.

Bertrand Lévy, « Géographie, mythe, conte, archétype : une introduction » in Le Globe, tome 154, 2014, p. 5-16.

Michel Maffesoli, Écosophie : Une écologie pour notre temps, Paris, Éditions du Cerf, 2017.

- - L'Ordre des choses : penser la postmodernité, Paris, CNRS, 2014. 
Identités de Pandore : à la rencontre du paysage comme image de soi

- - La Galaxie de l'Imaginaire : dérive autour de l'cuvre de Gilbert Durand, Paris, Berg International, 1980.

Carolyn Merchant, The death of nature: Women, ecology, and the scientific revolution. New York, Harper \& Row, 1980.

Mercedes Montoro Araque, Gautier, au carrefour de l'âme romantique et décadente, New York, Peter Lang, 2018.

Erich Neumann, «The Meaning of the Earth Archetype for Modern Times » in Princeton, 1994(1953), p. 165-226.

Dora et Erwin Panofsky, La Boite de Pandore. Les Métamorphoses d'un symbole mythique. Traduit de l'anglais par Maud Sissung, Vanves, Hazan, 2014.

Jean-Claude Schmitt, Ève et Pandora. La création de la femme, Paris, Gallimard, 2001.

Maxime Sheets-Johnstone, The Corporeal Turn. An Interdisciplinary Reader, Charlottesville-Exeter, Imprint Academic, 2009.

Jean-Pierre Vernant, Pandora, la première femme, Paris, Bayard, 2006.

- - «Les semblances de Pandora », in Blaise, F., Judet de La Combe, P., \& Rousseau, P. (Eds.), Le métier du mythe : Lectures d'Hésiode, Villeneuve d'Ascq, Presses universitaires du Septentrion, 1996, p. 381-392.

\section{NoTES}

1. Extrait de l'exemplaire de la Bibliothèque Nationale de France, Charles Chaisneau, Pandore, poème en trois chants, Paris, Artus-Bertrand, 1808, p. 29.

2. L'imaginaire comporte une dimension performative dans la mesure où il naît des représentations symboliques (Wunenburger, 2016) et des expériences corporelles. Voir Claude Fintz, Les imaginaires du corps en mutation: du corps enchanté au corps en chantier, Paris, L'Harmattan, 2005.

3. Erwin \& Dora Panofsky, La Boîte de Pandore. Les Métamorphoses d’un symbole mythique. Traduit de l'anglais par Maud Sissung, Vanves, Hazan, 2014, p. 53.

4. Ibid., p. 54.

5. Henri Estienne, L'introduction au Traité de la conformité des merveilles anciennes avec les modernes, Paris, 1556 , p. 5.

6. Maurice Scève, Délie, dizain II, 1544. Extrait de Lisa Wajeman, «Création de la femme, invention de la peinture. Eva prima Pandora, un tableau de Jean Cousin », in Jean-Claude Schmitt, Ėve et Pandora. La création de la première femme, Paris, Gallimard, 2001, p. 176.

7. Gaston Bachelard, La Poétique de l'espace, Paris, PUF, 1957, p. 161.

8. Ibid.

9. Ces archétypes - femme-eau, femme-plante, femme-terre et femme-rocher-, sont largement définis dans la thèse de doctorat « La féminisation du paysage. Décadentisme et postmodernité : vers une notion d'archétype » qui est en cours de préparation à l'Université de Grenade, sous la direction de Mercedes Montoro Araque, en adhésion à MITEMA (Mitos, imaginarios, temáticas pluridisciplinares) et au laboratoire Observatorio de Prospectiva Cultural (HUM-584).

10. Très proche de la pensée bachelardienne ainsi que de la théorie de l'imaginaire de Pierre Solié et Henri Corbin, l'Analyse des Mythes Corporels (AMC) est une technique d'art-thérapie s'appuyant sur les contes et les mythes pour explorer l'inconscient dans le champ des arts plastiques.

11. Csilla Kemenczei. Le féminin de la femme. «Rencontre Féminin-Masculin, regards croisés : MarieLouise von Franz - Pierre Solié », France, Association Autour de Marie-Louise von Franz, 2010.

12. Ibid.

13. Erich Neumann, «The Meaning of the Earth Archetype for Modern Times » in Princeton, 1994 (1953), p. 165-226.

14. Corin Braga, Archétypologie postmoderne. D'Edipe à Umberto Eco, Paris, Honoré Champion Éditeur, p. 16. 
15. Gilbert Durand, Introduction à la mythodologie. Mythes et sociétés, Paris, Albin Michel, 1996, p. 185.

16. Jean-Pierre Vernant, La première femme, Paris, Bayard, 2006, p. 5.

17. Jean-Pierre Vernant, « Les semblances de Pandora » in Blaise, F., Judet de La Combe, P., \& Rousseau, P. (Eds.), Le métier du mythe : Lectures d'Hésiode. Villeneuve d'Ascq, Presses universitaires du Septentrion, 1996, p. 383.

18. Ibid.

19. Hésiode, Les Travaux et les jours, v. 58, p. 88.

20. Florence Godeau, « Le vaisseau Pandore : histoire d'une traversée, de l'Europe vers l'Amérique (sur Henry James, Pandora, 1884) », Hg. von Heinz-Peter Preußer, Françoise Rétif und Juliane Rytz. Pandora. Zur mythischen Genealogie der Frau. Pandore et la généalogie mythique de la femme, Seiten und 16 Abbildungen auf 8 Tafeln, 2012, p. 181.

21. Gilbert Durand, Les Structures Anthropologiques de l'imaginaire : introduction à l'archétypologie générale, Paris, Dunod, 2016, p. 90.

22. Éliane Chiron, Paysages croisés. La part du corps, Paris, Publications de la Sorbonne, 2009, p. 10.

23. Gilbert Durand, Les Structures Anthropologiques de l'imaginaire, p. 104.

24. Ibid.

25. Gilbert Durand, Les Structures Anthropologiques de l'imaginaire, p. 94.

26. Hésiode, Les Travaux et les jours, v. 58-82, p. 88-89.

27. Gaston Bachelard, La Poétique de l'espace, p. 17.

28. Ibid., p. 92.

29. Ovide, Les Métamorphoses, Paris, Flammarion, 1966, p. 43.

30. «La verdure des bois et celle des prairies ; l'or flottant de la plaine et l'ambre des coteaux ; et l'émail des vallons, et le cristal des eaux ; et ces antres si frais, et ces bosquets si sombres; les jeux de la lumière et le repos des ombres, cet aspect varié d’objets intéressants. Charma, sans les blesser, leurs organes naissants, et sans peine introduite au fond de le pensé, leur image y resta légèrement tracée». Voir Charles Pierre Colardeau, Les hommes de Promethée, Paris, Le Jay, 1775, p. 24.

31. La version de Dante Gabriel Rosetti présente la devise « Nescitur ignescitur » (née des flammes) sur le devant de la boîte de Pandore évoquant l'argile cuite dont elle est issue. Cependant, dans le tableau de Watts Dunton, l'inscription est «Ultima manet spes », et les figures spirituelles formées dans la fumée qui s'échappe de la boîte rappellent les figures tristes des sonnets de Vain virtues et Pandora, écrits en 1870 par Rosetti pour accompagner son œuvre picturale.

32. Dans le tableau de John Williams Waterhouse, une Pandore solitaire, au milieu d'une forêt sombre et inaccessible, ouvre le luxueux coffre qui représente la transformation et le progrès. Ouvrir la boîte est dangereux, car l'évolution implique la rupture et la destruction, mais aussi l'entrée dans le monde chaotique des signes. Le garder fermé, c'est vivre dans la stabilité de la stagnation, en ignorant le monde dans lequel il réside.

33. Voir Johann Wolfgang Goethe, (1807-1808), « Pandora », traduction de Jean Tardieu, in Théatre complet, Paris, Gallimard.

34. Voir Honoré Balzac. (1854). Le député d'Arcis. Euvres complètes de Balzac. Scènes de la vie politique. Paris, Librairie Nouvelle. 1859. «Alors, puisque c'est ta femme, tu devrais bien la faire poser pour notre ami Dorlange, qui médite en ce moment une Pandore. Jamais il ne trouvera un modèle aussi magnifique » (Balzac 1859, p. 287). Dans l'imaginaire de Balzac, la femme Pandore se dévoile en tant qu'archétype, non par la terreur, mais à cause de l'admiration, au point d'être illustrée par une sculpture, le support d'un modèle parfait : "Quelques mois plus tard, au Salon de 1837 (...) j'avais exposé une statue qui fait quelque sensation. Autour de ma Pandore, constamment il y avait foule ». Ibid., p. 194.

35. Erwin \& Dora Panofsky, La Boîte de Pandore, p. 90.

36. Alain Roger, Court traité du paysage, Paris, Gallimard, 1997.

37. Ibidem., p. 17.

38. Michel Collot, Paysage et Poésie, du romantisme à nos jours, Paris, José Corti, 2005, p. 199. 
Identités de Pandore : à la rencontre du paysage comme image de soi

39. Ibid.

40. Gilbert Durand, Figures mythiques et visages de l'cuvre: de la mythocritique à la mythanalyse, Paris, Dunod, 1992, p. 245.

41. "Plus que celle de Prométhée ou que celle de Dionysos, c'est la figure d'Hermès qui acquière en notre temps la plus grande prégnance symbolique, car Hermès est justement figure de 'récurrence', dieu des carrefours et du commerce certes, mais surtout divinité des 'bornes' ». Michel Maffesoli, La Galaxie de l'Imaginaire : dérive autour de l'œuvre de Gilbert Durand. Paris, Berg International, 1980, p. 83.

42. Voir Gilbert Durand, Figures mythiques et visages de l'cuvre : de la mythocritique à la mythanalyse, Paris, Dunod, 1992, p. 68.

43. Gilbert Durand, Champs de l'imaginaire, Grenoble, Ellug, p. 230.

44. Ces constellations d'images comprennent les mythes, il s'agit de « couches 'génératives' et redondantes (synchroniques, paradigmatiques, constellations ou 'paquets' d'images, de mythèmes, 'images obsédantes', etc.) qui constituent la métalinguistique du sermo mythicus». Gilbert Durand, Champs de l'imaginaire, p. 117.

45. Hésiode, Les Travaux et les jours, v. 58-82, p. 724.

46. Voir Rothstein, B. (1972). Jean-Marie Domenach et Ivan Illich. «Un certain regard ». France, Office National de Radiodiffusion-Télévision Française (ORTF).

47. À l'origine, l'attribut de Pandore nétait pas une caisse, mais une jarre d'aspect maléfique déterminé par la curiosité féminine, de même que la pomme d'Ève. Au XVIe siècle, Érasme de Rotterdam confond le mythe de Pandore avec celui de Psyché, transformant la pithos (amphore) originale en pyxis (boîte). De cette erreur s'est imposée l'expression populaire de «la boîte de Pandore ».

48. Jean-Pierre Vernant, La première femme, Paris, Bayard, 2006, p. 61.

49. Voir Blanca Solares, Madre terrible. La diosa en la religión del México antiguo, Barcelone, Anthropos, 2007.

50. Voir Naomi Goldberg, Changing of the gods: feminism an the end of traditional religious, Boston, Beacon Press, 1979.

51. Voir Victor Hugo, Les Contemplations, VI, Au Bord de l'infini, Pleurs dans la nuit, IX, 1856.

52. Maxine Sheets-Johnstone propone une compréhension de notre existence corporelle en termes de mélodie cinétique, « as sheer physicality in movement ». Maxine Sheets-Johnstone, The Corporeal Turn. An Interdisciplinary Reader, Charlottesville, Exeter, Imprint Academic, 2009, p. 45.

53. Rothstein, B. Jean-Marie Domenach et Ivan Illich. «Un certain regard».

54. Patrice Guillamaud, «L'essence du kairos » in Revue des Études Anciennes. Tome 90, 1988, n 3-4, p. 371.

55. Bertrand Lévy, " Géographie, mythe, conte, archétype : une introduction » in Le Globe, tome 154, 2014, p. 11.

56. Jean-Louis Benoit, J. 2012. « La Vierge Marie dans les Miracles de Notre-Dame, créature féerique et mère de Dieu » in Anna Caiozzo, Femmes médiatrices et ambivalentes : Mythes et imaginaires. Paris, Armand Colin, 2012, p. 120

57. Karl Taube, Mythes aztèques et mayas, Paris, Éditions du Seuil, 1995, p. 85

58. Michel Cazenave, Visages du fémin sacré, Paris, Éditions Entrelacs, 2012, p. 68-69.

59. Pierre Hodot, Le voile d'Isis : Essai sur l'bistoire de l'idée de Nature, Paris, Folio, 2004, p. 6.

60. Juan Eduardo Cirlot, Diccionario de simbolos tradicionales. Barcelone, Luis Miracle, 2004, p. 305.

61. Pierre Hodot, Le voile d'Isis : Essai sur l'histoire de l'idée de Nature, p. 241-242, note 17, p. 359 ; Macrobe, Saturnales, I, 20, 18.

62. Gilbert Durand, Champs de l'imaginaire, p. 39.

63. Françoise D’Eaubonne, Écologie et féminisme : Révolution ou mutation ?, Paris, Libre \& Solidaire, 1978, p. 15.

64. Françoise D'Eaubonne, Le féminisme ou la mort, Paris, Pierre Horay, 1974, p. 252.

65. Pour Michel Maffesoli, la simple raison se voir complétée par « les sens, les instincts, les sentiments et d'autres manifestations de cette capacité de fantasmer constituant l'entiérété de l'humain-nature » (Michel Maffesoli, L'Ordre des choses : penser la postmodernité, Paris, CNRS, 2014, p. 35). 
66. Jean-Pierre Vernant, La première femme, Paris, Bayard, 2006, p. 73.

67. "The metaphor of the earth as a nurturing mother was gradually to vanish as a dominant image as the Scientific Revolution proceeded to mechanize and to rationalize the world view ». Carolyne Merchant, The death of nature: Women, ecology, and the scientific revolution, New York, Harper \& Row, 1980, p. 2.

68. "As woman's womb had symbolically yielded to the forceps, so nature's womb harbored secrets that through technology could be wested from her grasp for use in the improvement of the human condition ». Carolyne Merchant, The death of nature: Women, ecology, and the scientific revolution, p. 169.

69. Rothstein, B. Jean-Marie Domenach et Ivan Illich. «Un certain regard».

70. Michel Maffesoli, L'Ordre des choses : penser la postmodernité, p. 34.

71. Ibid.

72. Augustin Berque, La pensée paysagère, Paris, Archibooks, 2008, p. 9.

73. Ibid., p. 89.

74. Pierre Lévêque, « Pandora ou la terrifiante féminité », in Kernos, 1, 1988, p. 60

75. Ibid., p. 58.

76. Philon d'Alexandrie, De aeternitate mundi, XII, 63.

77. Gilbert Durand, «Le symbolisme de la terre », in Encyclopaedia Universalis, 1972.

78. Platon, Ménexène ou l'oration funèbre, 238b.

79. Gilbert Durand, Les Structures Anthropologiques de l'imaginaire : introduction à l'archétypologie générale, p. 242. 